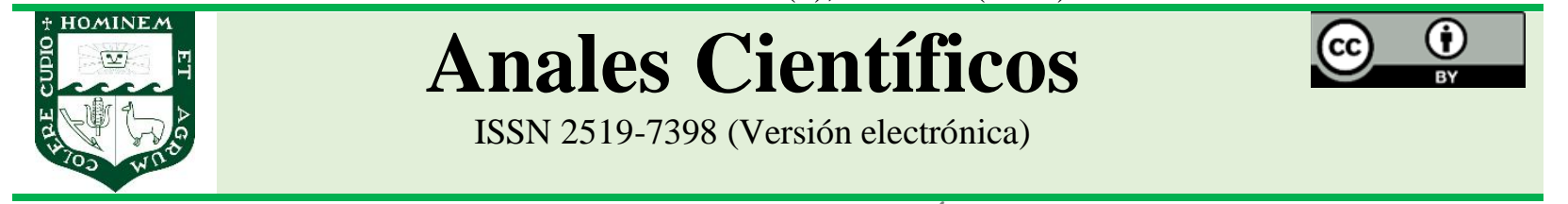

ARTÍCULO ORIGINAL - RESEARCH ARTICLE http://dx.doi.org/10.21704/ac.v82i1.1755

\title{
ELABORACIÓN DE SNACK EXTRUIDO A PARTIR DE CEREALES Y CONCENTRADO DE PROTEÍNA DE POTA (Dosidicus gigas) Y DETERMINACIÓN DE SU VIDA ÚTIL
}

\section{Extruded snack elaboration from cereals and giant squid protein concentrate (Dosidicus gigas) and determination of its shelf life}

\author{
Karina Espinoza C. ${ }^{1}$; David Roldan A. ${ }^{1}$; ; Nancy Martínez O. ${ }^{1 *(1)}$ \\ ${ }^{1}$ Facultad de Pesqueria, Universidad Nacional Agraria La Molina, La Molina, 15024, Lima, Perú. \\ *E-mail:nmo@lamolina.edu.pe
}

Recibido: 08/09/2020; Aceptado: 30/06/2021; Publicado:31/08/2021

\begin{abstract}
The objective was to obtain a snack extruded from cereals (corn, rice, kiwicha) and giant squid protein concentrate (CPCG), which meets the protein and amino acid requirements for children aged 5 to 12 years. The process flow was reception of the raw material, ground, weighed and mixed, conditioning the mixture, extruded, cooled, packed in a bioriented polypropylene bag, sealed and stored. The base mix included: $60.0 \%$ corn, $18.5 \%$ rice, $15.0 \%$ kiwicha and 4.0\% CPCG. Likewise, the suitable parameters for the extrusion process were: $12.6 \%$ moisture of the mixture and screw speed of the extruder of 150 RPM. The extruded snack had a round shape with an average diameter of $1,3 \mathrm{~cm}$, cream color and no smell and taste of the giant squid resource. The expansion index was 3.3 and the apparent density was $66.76 \mathrm{~g} / \mathrm{L}$. Its chemical composition was $16.8 \%$ protein, $5.35 \%$ moisture, $1.89 \%$ fat, $1.05 \%$ fiber, $2.01 \%$ ash, and $72.91 \%$ carbohydrates. The extruded snack presented microbiological values in accordance with those required by RM N $591-2008 / M I N S A$. Its shelf life, determined by Accelerated Testing, was 15 weeks. Nutritionally, the extruded snack can be used for children.
\end{abstract}

Keywords: snack; extruded; cereals; giant squid; shelflife

\section{RESUMEN}

El objetivo fue obtener un snack extruido a partir de una mezcla de cereales (maíz, arroz, kiwicha) y concentrado de proteínas de pota (CPCG), que cumpla con los requerimientos de proteínas y aminoácidos para niños de 5 a 12 años. El flujo de proceso utilizado fue: recepción de la materia prima, molido, pesado y mezclado, acondicionado de la mezcla, extruido, enfriado, envasado en bolsa de polipropileno biorientado, sellado y almacenado. La mezcla base incluyó: 60,0\% de maíz, 18,5\% de arroz, 15,0\% de kiwicha y 4,0\% de CPCG. Asimismo, los parámetros adecuados para el proceso de extrusión fueron: humedad de la mezcla mínima de 12,6\% y velocidad de tornillo de la extrusora de 150 RPM. El snack extruido presentó forma redonda con un diámetro promedio de 1,3 cm, color crema y ausencia de olor y sabor al recurso pota. El índice de expansión fue de 3,3 y la densidad aparente de 66,76 g/L. Su composición química fue: proteína $16,8 \%$, humedad 5,35\%, grasa $1,89 \%$, fibra $1,05 \%$, ceniza $2,01 \%$ y carbohidratos $72.91 \%$. El snack extruido presentó valores microbiológicos conformes a lo exigido por la RM N591-2008/MINSA. Se determinó que la vida útil, según pruebas aceleradas, fue de 15 semanas. Nutricionalmente el snack extruido puede ser destinado a la población infantil. 


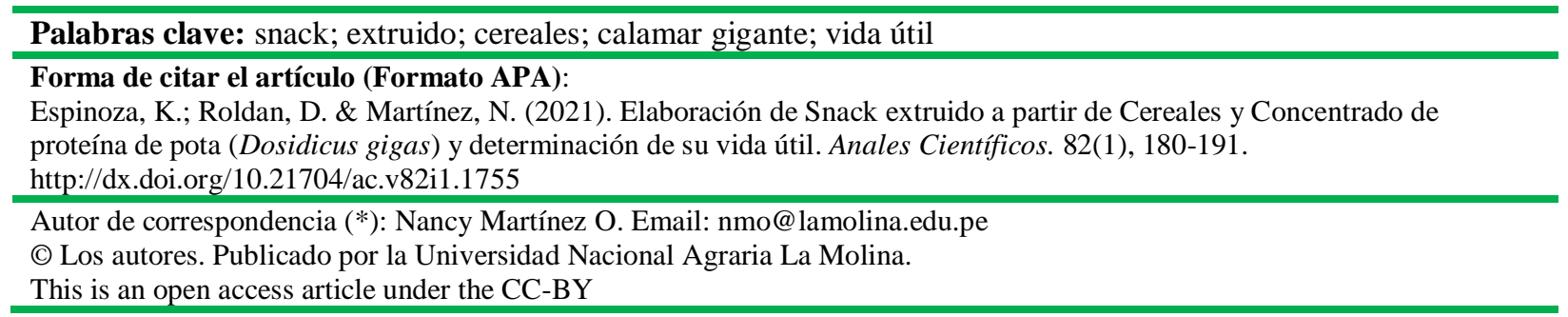

\section{INTRODUCCIÓN}

Las nuevas tecnologías buscan mejorar los procesos de elaboración de productos alimenticios que tengan características funcionales para el consumo humano. Los cereales actualmente son utilizados en procesos de extrusión, proceso que viene desarrollándose con bastante éxito (Osorio, 2009). En el Perú existen muchos recursos que se pueden utilizar para elevar su contenido de aminoácidos esenciales que les permitan a los cereales alcanzar una base nutritiva más importante, entre ellos se encuentran los granos andinos y proteínas de origen animal como es el caso de los recursos marinos.

El proceso de extrusión de cereales y granos andinos es una alternativa adecuada para la alimentación y nutrición de niños, sin embargo, las mezclas utilizadas pueden ser mejoradas nutricionalmente con la adición de proteínas de origen animal. Por otro lado, el concentrado de proteína de pota (Dosidicus gigas) presenta adecuadas características nutricionales y sensoriales (Lazo, 2006), que podría ser incluido en las formulaciones de estos extruidos con la finalidad de obtener un producto funcional que cumpla con los requerimientos de alimentación infantil.

La pota se considera una buena fuente de proteínas de alto valor biológico que contiene todos los aminoácidos esenciales. Su contribución en grasas es baja, así como su aporte en calorías, además contiene vitaminas $\mathrm{B}$, fundamentales para el metabolismo, el mantenimiento del sistema nervioso central, del aparato digestivo y la piel. Presenta minerales como el fósforo, potasio y magnesio, que ayudan a la formación de huesos y dientes. Por otro lado, la pota contiene Taurina, un aminoácido que regula la presión sanguínea disminuye la formación de coágulos en las venas, mejora la visión y visibilidad nocturna y actúa como antioxidante (Córdova, 2016).

El concentrado de proteína de pota es un producto obtenido utilizando sólo la fracción muscular; es elaborado para consumo humano directo, presenta un valor biológico del $90 \%$ con un contenido de $86 \%$ de proteína y $3 \%$ de grasa ( $40 \%$ de las cuales son omega 3). Se elabora sin el uso de solventes ni agentes químicos de ninguna especie (Lazo, 2006).

Los cereales son semillas de plantas y en la mayor de parte de los casos, aunque con algunas excepciones, pertenecen a la familia de las gramíneas. Al igual que todas las semillas, los cereales tienen gran aporte nutricional ya que contienen todos los nutrientes que el embrión de la planta necesita para empezar a crecer, en este grupo se encuentran el arroz, maíz, cebada, kiwicha, entre otros (Muller y Tobin, 1986).

Los snacks son productos alimenticios, salados o dulces, fritos o extruidos, que no han sido sometidos a la acción de leudantes químicos o biológicos, que se presentan en diferentes formas y generalmente son envasados (ITINTEC, 1984).

La extrusión, es definida como el proceso que consiste en dar forma a un producto, forzándolo a través de una abertura con diseño específico. La extrusión puede o no implicar simultáneamente un proceso de cocción (Valls, 1993). La tecnología de la extrusión se destaca en la industria de alimentos como un proceso eficiente, utilizándose en el procesamiento de cereales y proteínas, para alimentación humana y animal (Cugiño, 2008).

El proceso de extrusión a altas temperaturas (100$\left.180{ }^{\circ} \mathrm{C}\right)$ y presión por corto tiempo (30-120 segundos), es uno de los procesos tecnológicos de mayor versatilidad para la elaboración de productos 
alimenticios a partir de cereales y leguminosas (Delahaye et al., 1997). Su alta productividad, bajos costos de operación, eficiencia energética, obtención de productos de alto valor biológico y elevada digestibilidad de las proteínas (Mercier, 1993; Milán-Carrillo et al., 2002) lo convierten en un proceso tecnológico atractivo. Así mismo, Yanova et al. (2019) mencionan la mejora de la eficiencia económica de las empresas procesadoras de cereales mediante la introducción de tecnologías desarrolladas de extrusión y producción de harina a partir de granos extruidos de los principales cultivos de cereales.

Después de la elaboración del extruido se aseguró que su calidad se mantenga en el nivel requerido de sus cualidades nutricionales y organolépticas, durante el almacenamiento y distribución por lo cual se determinó su vida útil utilizando la metodología de pruebas aceleradas según la cinética de reacciones de deterioro.

Por lo anterior, el objetivo de la investigación fue elaborar un snack extruido de cereales, granos andinos y concentrado de proteína de pota, que cumpla con los requerimientos de proteína y aminoácidos para la población infantil (5 a 10 años), de buena aceptabilidad y determinar su vida útil, utilizando pruebas aceleradas

\section{MATERIALES Y MÉTODOS}

La investigación se desarrolló en los laboratorios de la Universidad Nacional Agraria La Molina y en la planta de la empresa CERECROCKS. Se utilizó una extrusora artesanal de alimentos de tornillo simple con sistema de alimentación por gravedad.

La evaluación sensorial del concentrado de pota se realizó según la tabla de análisis sensorial reportada por Lazo (2006); para la harina de quinua, harina de kiwicha, harina de arveja y maíz molido, se solicitó al proveedor el certificado de calidad, además se realizó una inspección de los siguientes factores: hermeticidad del envase, olor, color y sabor del producto. La granulometría de las materias primas se determinó según el sistema de mallas Retsch, sugeridas por Perry (1980). En el producto extruido se determinó el indice de expansión (IE) según Kameko (2005) y la densidad aparente (DA), para ello se llenó una probeta graduada con un volumen conocido y adiciona una cantidad de muestra también conocida. Se aplicó la fórmula universal para el cálculo de densidad. Los análisis de la composición química proximal de la materia prima y producto final se realizaron según los procedimientos propuestos por la Association of Official Analytical Chemestry (AOAC, 2012).

El análisis microbiológico se realizó siguiendo las recomendaciones dadas por Ingram et al. (1983); los análisis realizados fueron los exigidos por la RM $\mathrm{N}^{\circ}$ 591-2008/MINSA para los productos cocidos de consumo directo como extruidos, expandidos, hojuelas instantáneas y otros similares. Las muestras extruidas experimentalmente fueron evaluadas sensorialmente mediante pruebas de preferencia utilizando un panel de 20 personas sin entrenamiento.

Los formatos para la Prueba de Preferencia fueron elaborados según recomendación de Ureña et al. (1999) y los resultados se procesaron de acuerdo con el método estadístico de Friedman con un nivel de significancia del 5\% (Conover, 1980), de haber diferencia entre las muestras se procederá con la prueba estadística de comparaciones múltiples de Wilcoxon. El producto final fue evaluado sensorialmente utilizando un panel semientrenado de 10 personas. Se utilizó el método no paramétrico de la prueba de Friedman. Los resultados se procesaron con el programa estadístico InfoStat 08. La determinación de la vida útil del snack extruido fue realizada mediante la cinética de deterioro por pruebas aceleradas (ASLT), para ello las muestras del snack extruido fueron almacenadas durante 10 semanas a temperaturas de $35^{\circ} \mathrm{C}, 45^{\circ} \mathrm{C}$ y $50^{\circ} \mathrm{C}$ con $85 \%$ de HR, valor sugerido en pruebas de estabilidad de cereales (Fernández et al., 2009). Cada semana se determinó la humedad y se evaluó sensorialmente las muestras con la prueba de valoración de calidad de Karlsruhe (Wittig, 1981) adaptado para el producto y fue realizado por 8 jueces. Se consideró a la humedad como el indicador de deterioro (Kilcast y Subramaniam, 2000). La humedad crítica fue determinada cuando la calidad total fue de 5,5 según la valoración de calidad de Karlsruhe. 
La Figura 1 muestra el flujo general utilizado para elaborar el snack extruido a partir de cereales, granos andino y CPCG, elaborado en base las recomendaciones reportadas por Sevatson y Huber (2000), para la producción de cereales para desayuno directamente expandidos. Las materias primas fueron recepcionadas en bolsas herméticas selladas. Fueron pesadas según las mezclas determinadas en el cómputo químico. La mezcla de las materias primas se realizó por 5 minutos, posteriormente se determinó la humedad de la mezcla y se procedió a la extrusión. El proceso de extrusión se realizó utilizando $5 \mathrm{~kg}$ de cada mezcla, la muestra extruida fue enfriada en condiciones ambientales y envasada a granel en bolsas de polietileno de baja densidad. El producto final fue envasado en bolsas de polipropileno biorientado (BOPP) metalizado de alta barrera, y cerrado utilizando una selladora de mordaza.

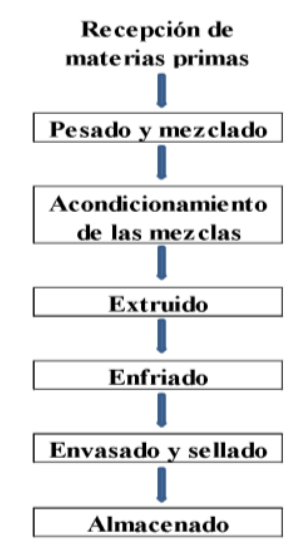

Figura 1. Flujo general para la elaboración de un snack extruido.

El estudio se realizó en cuatro etapas: (1) Selección de cuatro mezclas teóricas para elaborar el snack extruido. Se diseñaron seis mezclas teóricas $\left(\mathrm{MT}_{1}\right.$, $\mathrm{MT}_{2}, \mathrm{MT}_{3}, \mathrm{MT}_{4}, \mathrm{MT}_{5}$ y $\mathrm{MT}_{6}$ ), de las cuales se seleccionaron cuatro, para ser extruidas. Para la selección de las cuatro mezclas se tuvo en cuenta la cantidad de proteína de la mezcla y el score de aminoácidos, según procedimiento reportado por Young y Reeds (2002). Se usó la referencia de FAO (1981) y como patrón los requerimientos reportados por la norma NTP-209.260 (INDECOPI, 2004). (2) Determinación de la humedad adecuada para la extrusión. Inicialmente, en las cuatro mezclas seleccionadas, se determinó la humedad de la mezcla antes del proceso de extrusión; posteriormente, mediante prueba de preferencia se seleccionó dos de ellas. En estas mezclas seleccionadas se modificaron los contenidos de humedad antes de la extrusión. Las nuevas mezclas fueron codificadas como $\mathrm{MH}_{1}, \mathrm{MH}_{2}, \mathrm{NH}_{1}$ y $\mathrm{NH}_{2}$. Se realizó una prueba de preferencia para determinar la mejor humedad de la mezcla extruida (3) Determinación de la velocidad adecuada del tornillo de la extrusora. La formulación de la mezcla del experimento anterior fue sometida a un proceso de extrusión con tres diferentes velocidades de tornillo. Las velocidades que se ensayaron fueron: 160 (V1), 180 (V2) y 150 (V3) RPM. (4) Para determinar la vida útil del producto, se utilizó la cinética de reacciones de deterioro. Primero se obtuvo el orden de reacción evaluando la variación de la humedad a través del tiempo de almacenamiento. Según el orden de reacción se aplicó la ecuación de cinética correspondiente.

$$
\frac{d A}{d t}=-k(A)^{n} \quad \text { Ec. } 1
$$

Para el orden cero se aplica la ecuación:

$$
\mathrm{A}=\mathrm{A}_{\mathrm{o}}-\mathrm{kt} \quad \text { Ec. } 2
$$

Siendo A, el indicador de deterioro al tiempo t, A0 es indicador de deterioro al tiempo cero, $\mathrm{k}$ es la velocidad de la reacción y n es el orden de reacción, (Labuza, 1982).

Se utilizó la ecuación de Arrhenius

$$
\mathrm{k}=k_{o} e^{\left[-\frac{E_{A}}{R T}\right]} \quad \text { Ec. } 3
$$

Donde:
ko: Factor pre-exponencial
EA: Energía de activación $(\mathrm{KJ} / \mathrm{mol})$
$\mathrm{R}$ : Constante universal de los gases $(0,008314 \mathrm{KJ} / \mathrm{mol} \mathrm{K})$
$\mathrm{T}$ : Temperatura absoluta (K)

Luego de conocer los parámetros cinéticos se calculó la vida útil del producto.

\section{Análisis microbiológico}

Se realizaron las pruebas microbiológicas al producto, siguiendo los criterios microbiológicos de la norma nacional RM N591-2008/MINSA y se 
utilizó la metodología de la Comisión Internacional de estándares microbiológicos de los alimentos (Ingram et al., 1983).

\section{RESULTADOS Y DISCUSIÓN}

Análisis físico sensorial de las materias primas. La evaluación sensorial del CPCG, según la tabla propuesta por Lazo (2006), obtuvo 12 sobre 12 puntos. El color fue blanco lechoso, atribuido a un proceso de deshidratación adecuado y controlado teniendo especial atención a que no se genere la reacción de Maillard. El sabor fue neutro y el olor fue ligero a marisco. Roldán y Lazo (2009) indican que estas características pueden pasar desapercibidas fácilmente al mezclarse con otros ingredientes en caso de ser utilizado como insumo para alimentos enriquecidos.
Los resultados de la granulometría indicaron que el CPCG ha recibido una molienda muy fina, ya que el mayor porcentaje de la muestra $(63,75 \%)$ atravesó el tamiz de malla 100, similares características presentaron las harinas de quinua, kiwicha y arveja. La Harina de maíz presentó una granulometría de 0,5 mm de diámetro en el $90 \%$ de sus partículas Harper (1981) explica que en extrusoras monotornillo (como el usado en la investigación) las partículas muy finas no son adecuadas porque se funden rápidamente y la masa resultante no tiene un buen transporte.

Análisis químico proximal de las materias primas. Los resultados de los análisis de composición proximal realizados a las materias primas se muestran en la Tabla 1.

Tabla 1. Análisis químico proximal de materias primas utilizadas.

\begin{tabular}{lccccc}
\hline Componente & CPCG (\%) & $\begin{array}{c}\text { Harina de } \\
\text { maíz }(\boldsymbol{\%})\end{array}$ & $\begin{array}{c}\text { Harina de Quinua } \\
(\boldsymbol{\%})\end{array}$ & $\begin{array}{c}\text { Harina de } \\
\text { Kiwicha (\%) }\end{array}$ & $\begin{array}{c}\text { Harina de Arveja } \\
(\%)\end{array}$ \\
\hline Proteínas & 85,42 & 8,2 & 13,41 & 14,98 & 22,4 \\
Grasas & 2,65 & 1,9 & 5,9 & 7,46 & 0,98 \\
Cenizas & 4,84 & 2,9 & 2,6 & 2,84 & 2,5 \\
Humedad & 5,34 & 14,5 & 11,65 & 1,2 & 10,5 \\
\hline
\end{tabular}

El contenido de proteínas del CPCG fue similar al reportado por Lazo (2006) y Osorio (2009), las pequeñas diferencias pueden ser atribuidas a que la cantidad de proteínas depende del proceso de producción, grado de frescura y cantidad de lípidos de la materia prima, según reporte de Madrid et al. (1994). El alto contenido de proteína en el CPCG $(85,42 \%)$ nos sugiere que con el uso de pequeñas cantidades se podría satisfacer las necesidades de proteínas en la dieta diaria, y sería posible su uso como fuente de proteína animal en la formulación de un producto funcional para consumo humano. Es de entender que se deben encontrar mezclas de alimentos para consumo humano donde no predomine exageradamente ningún aminoácido esencial, debido a efectos nocivos por una especie de antagonismo entre estos mencionados que originan un balance de nitrógeno negativo por pérdidas de proteínas (García, 1990).

Selección de cuatro mezclas teóricas para elaborar el snack extruido. En la Tabla 2 se muestran las mezclas $\left(\mathrm{MT}_{1}, \mathrm{MT}_{2}, \mathrm{MT}_{3}, \mathrm{MT}_{4}, \mathrm{MT}_{5}\right.$ y $\left.\mathrm{MT}_{6}\right)$ propuestas tomando en consideración el cumplimiento de los requerimientos de aminoácidos para consumo infantil según NTP-209.260 (2004). Así mismo la Tabla 2 señala la cantidad proteica de la mezcla, el aporte calórico, el aminoácido en menor cantidad (aminoácido limitante) y score de aminoácidos. 
Tabla 2. Formulaciones teóricas propuestas.

\begin{tabular}{cccccccccccc}
\hline $\mathbf{F}$ & $\begin{array}{c}\text { Polenta de } \\
\text { maíz }\end{array}$ & CPCG & $\begin{array}{c}\text { H. de } \\
\text { Quinua }\end{array}$ & $\begin{array}{c}\text { H. de } \\
\text { Kiwicha }\end{array}$ & $\begin{array}{c}\text { H. de } \\
\text { Arveja }\end{array}$ & Arroz & $\begin{array}{c}\text { Leche en } \\
\text { polvo }\end{array}$ & Proténa Total & Kcal/100 & $\begin{array}{c}\text { Aminóádo en } \\
\text { menor conc. }\end{array}$ & $\begin{array}{c}\text { Score } \\
\text { aminoácidos }\end{array}$ \\
\hline $\mathrm{MT}_{1}$ & 60,0 & 4,0 & 20 & 0 & 10 & 6,0 & 0 & 12,89 & 334,32 & Triptófano & 82,8 \\
$\mathrm{MT}_{2}$ & 60,0 & 4,0 & 15 & 0 & 6 & 15,0 & 0 & 12,31 & 335,40 & Triptófano & 85,5 \\
$\mathrm{MT}_{3}$ & 60,0 & 4,0 & 0 & 15 & 0 & 18,5 & 2,5 & 14,51 & 334,87 & Lisina & 96,7 \\
$\mathrm{MT}_{4}$ & 60,0 & 5,0 & 0 & 15 & 5 & 15,0 & 0 & 13,96 & 334,55 & Triptófano & 95,3 \\
$\mathrm{MT}_{5}$ & 60,0 & 5,0 & 0 & 13 & 0 & 22,0 & 0 & 13,14 & 335,60 & Lisina & 95,6 \\
$\mathrm{MT}_{6}$ & 60,0 & 5,0 & 8 & 7 & 10 & 10,0 & 0 & 14,09 & 334,67 & Triptófano & 89,3 \\
\hline
\end{tabular}

Ensayos previos demostraron que el uso de $10 \%$ de CPCG genera un producto extruido muy duro, atribuido a su elevado contenido de proteína, por ello solo se ensayaron dos cantidades en las mezclas (4\% y $5 \%$ ). La cantidad de maíz molido fue de $60 \%$ para todos los casos; basado en las recomendaciones de Harper (1981) quien recomienda utilizar no menos de $60 \%$ de maíz en las mezclas para extrusión. La inclusión de leche en polvo $\left(\mathrm{MT}_{3}\right)$ mejoró el contenido de aminoácidos y el cómputo químico en comparación con las otras mezclas, este aporte confirmó las bondades de este insumo como fuente de proteína animal.

El cómputo químico permitió determinar que los aminoácidos limitantes en las 6 mezclas propuestas fueron el triptófano $\left(\mathrm{MT}_{1}, \mathrm{MT}_{2}, \mathrm{MT}_{4}, \mathrm{y} \mathrm{MT}_{6}\right)$ y la lisina $\left(\mathrm{MT}_{3}\right.$ y $\left.\mathrm{MT}_{5}\right)$. Tanto la lisina como el triptófano son nutrientes importantes para la síntesis de proteína corporal, así como los demás aminoácidos esenciales; sin embargo, el score químico es superior a 80 en todos los casos; valor mínimo recomendado por la NTP 209.260 (INDECOPI, 2004), respecto al contenido de aminoácidos, de mezclas para consumo infantil. Por el mayor contenido de proteínas y el mejor score de aminoácidos se seleccionaron las siguientes mezclas teóricas: $\mathrm{MT}_{1}, \mathrm{MT}_{3}, \mathrm{MT}_{4}$ y $\mathrm{MT}_{5}$.

Tabla 3. Contenido de humedad (\%) de las mezclas seleccionadas.

\begin{tabular}{lcccc}
\hline \multirow{2}{*}{ Componente } & \multicolumn{4}{c}{ Formulaciones seleccionadas } \\
& $\mathbf{M T}_{\mathbf{1}}$ & $\mathbf{M T}_{\mathbf{3}}$ & $\mathbf{M T}_{\mathbf{4}}$ & $\mathbf{M T}_{\mathbf{5}}$ \\
\hline Humedad & 12,23 & 12,55 & 10,88 & 12,76 \\
\hline
\end{tabular}

El contenido de humedad de las mezclas teóricas seleccionadas $\left(\mathrm{MT}_{1}, \mathrm{MT}_{3}, \mathrm{MT}_{4}\right.$ y $\left.\mathrm{MT}_{5}\right)$, que fueron sometidas al proceso de extrusión, se reportan en la Tabla 3.

Según Mercier et al. (1998), el mínimo porcentaje de humedad de mezclas para la extrusión es de $10 \%$, en el caso de extrusoras de bajo costo, humedades mayores de $20 \%$ originan un resbalamiento del material dentro del cilindro. Según Gonzáles et al. (2002); el material debe poseer humedades que varíen entre 10 y $35 \%$.

Las cuatro mezclas seleccionadas $\left(\mathrm{MT}_{1}, \mathrm{MT}_{3}, \mathrm{MT}_{4}\right.$ y $\mathrm{MT}_{5}$ ) fueron consideradas adecuadas para ser extruidas, se utilizó 160 RPM de velocidad de tornillo y dado de forma cilíndrica de $0,4 \mathrm{~cm}$ de diámetro. Posteriormente los productos extruidos fueron evaluados sensorialmente según los atributos de sabor, olor, crocantes y aspecto. Los resultados indicaron que ninguna muestra de snack extruido obtuvo un resultado negativo respecto al olor; además de no reportarse ninguna semejanza con el olor al recurso pota.

De las pruebas estadísticas se observó que la crocancia que presentó el extruido fue la principal característica de evaluación, seguido del sabor.

Andersson et al. (1983) estudiaron las características organolépticas de los productos extruidos con respecto a la fibra y determinaron que a mayor cantidad de fibra se tiene un color más oscuro y sabor más amargo; también, los productos con alto contenido de fibra resultan poco expandidos. La Figura 2 muestra el porcentaje de preferencia de cada uno de los snacks extruidos. 


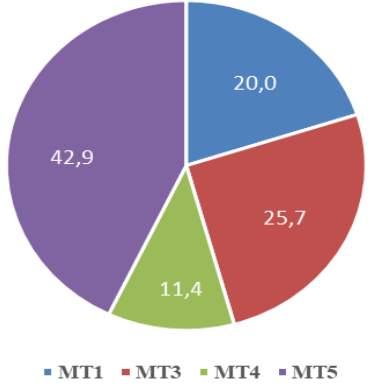

Figura 2. Preferencia según muestras extruidas.

La muestra extruida $\mathrm{MT}_{5}$ alcanzó la mayor preferencia $(42,9 \%)$ seguido de la muestra $\mathrm{MT}_{3}$ $(25,7 \%)$. Se concluyó que las dos muestras $\mathrm{MT}_{3} \mathrm{y}$ MT5 fueron apropiadas para la siguiente etapa de la investigación.
Determinación de la humedad adecuada para la extrusión. Para determinar la humedad adecuada se utilizaron las mezclas seleccionadas $\left(\mathrm{MT}_{3}\right.$ y $\mathrm{MT}_{5}$ ), se ensayaron dos porcentajes de humedad en cada una de ellas. En la Tabla 4 se detalla el valor de humedad experimental para las mezclas seleccionadas FT3 y FT5, así como la cantidad de agua que se debe agregar para preparar las nuevas muestras codificadas de la siguiente manera: MT3 = M y MT5 = N.

Las seis formulaciones (M, MH1, MH2, N, NH1, $\mathrm{NH} 2$ ) fueron extruidas y posteriormente evaluadas sensorialmente según los atributos de sabor, olor, crocancia y aspecto.

Tabla 4. Humedad experimental de las mezclas y cantidades de agua para agregar a las nuevas mezclas ensayadas.

\begin{tabular}{lcccccc}
\hline Concepto & $\mathbf{M}\left(\mathbf{M T}_{\mathbf{3}}\right)$ & $\mathbf{M H}_{\mathbf{1}}$ & $\mathbf{M H}_{\mathbf{2}}$ & $\mathbf{N}\left(\mathbf{M T}_{\mathbf{5}}\right)$ & $\mathbf{N H}_{\mathbf{1}}$ & $\mathbf{N H}_{\mathbf{2}}$ \\
\hline Humedad teórica & 12,55 & 12,60 & 13,60 & 12,76 & 13,30 & 14,30 \\
Humedad experimental (\%) & 10,60 & - & - & 12,30 & - & - \\
Cantidad de agua para & - & 91,53 & 138,88 & - & 46,13 & 93,34 \\
agregar (ml/5kg) & & & & &
\end{tabular}

Según los resultados, las diferencias entre la humedad teórica $(12,55 \%$ y $12,76 \%)$ ) y calculada $(12,60 \%$ y $13,30 \%)$ podría deberse a diferentes características, tales como: zona de cultivo, forma de extracción, temporada, etc.

La Figura 3 muestra los puntajes promedio obtenidos por atributos de los snacks extruidos (M, MH1, MH2, N, NH1, NH2).

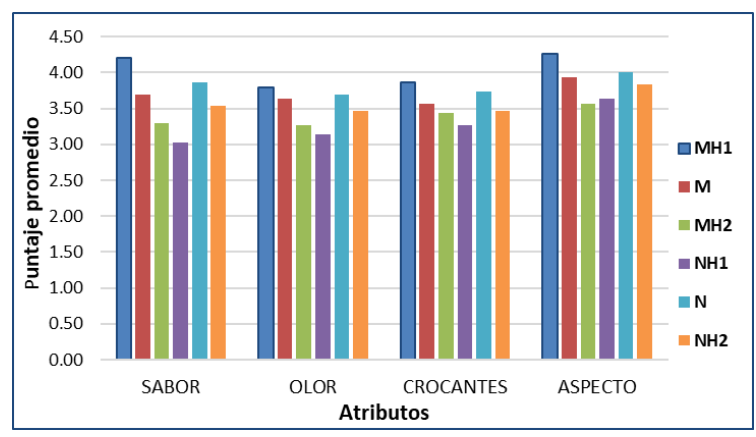

Figura 3. Puntaje obtenido respecto a los atributos evaluados de cada muestra extruida.
En la Figura 3 se observa que la muestra extruida MH1 fue la que recibió un mayor calificativo, siendo el aspecto el atributo que más agradó al panel. El que obtuvo el menor puntaje promedio fue el $\mathrm{NH} 1$, siendo el sabor el atributo que menos fue aceptado. Los resultados estadísticos de las seis muestras de snacks extruidos para cada atributo demostraron que todos ellos difieren significativamente. Así mismo la muestra MH1 obtuvo mayores calificativos de sabor, olor, crocantes y aspecto. Cabe destacar que ninguna obtuvo un calificativo negativo respecto al olor.

Respecto al sabor la muestra NH1 y MH1 presentaron un sabor ligeramente amargo debido a que al tener una mayor cantidad de agua el producto se quemó ligeramente en el proceso productivo. De las pruebas estadísticas se obtuvo que el atributo más importante en la prueba sensorial, según el factor humedad, fue la crocancia, seguida del aspecto.

En la Figura 4 se muestra el porcentaje de los panelistas que prefirieron cada una de las muestras 
de los snacks extruidas a partir de las formulaciones $\mathrm{M}, \mathrm{MH} 1, \mathrm{MH} 2, \mathrm{~N}, \mathrm{NH} 1$ y NH2

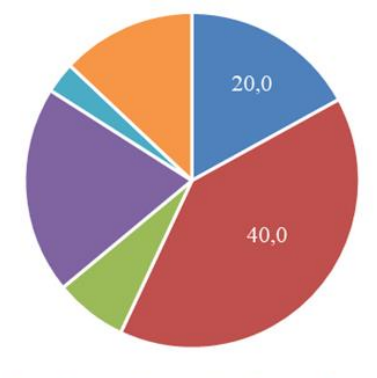

- M $=\mathrm{MH} 1=\mathrm{MH} 2 \cdot \mathrm{N}-\mathrm{NH} 1=\mathrm{NH} 2$

Figura 4. Porcentaje de preferencia por muestras evaluadas.

Los resultados del panel indicaron que la muestra extruida MH1 tuvo mayor grado de preferencia $(40 \%)$ seguido de la muestra N (20\%). Por ello la mezcla del snack extruido MH1 con $12,60 \%$ de humedad, fue seleccionada para la siguiente etapa de la investigación.

Determinación de la velocidad de tornillo para el proceso de extrusión. Se evaluaron tres diferentes valores de velocidad de tornillo para la mezcla MH1. Las velocidades aplicadas en el proceso de extrusión son: V1 (160 RPM), V2 (180 RPM) y V3 (150 RPM)

Los resultados estadísticos de la evaluación sensorial respecto de los atributos de las nuestras de snack extruido obtenidos con las diferentes velocidades del tornillo de la extrusora indicaron que la muestra V3 fue la que recibió un mayor calificativo, siendo el sabor el atributo que más agradó a los degustadores. La muestra V2 fue la que obtuvo el menor puntaje promedio, siendo la crocancia el atributo que menos se aceptó.

La muestra V3 fue la tuvo un sabor más agradable (maíz ligeramente dulce), en cambio la muestra V2 fue la que tuvo un sabor ligeramente diferente (ligeramente quemado), por medio de la Prueba de Wilcoxon, se corroboró que la muestra V3 y V2 tienen diferencias significativas. Respecto a la crocancia evaluada en los tres snacks extruidos, estos no presentaron diferencias significativas, según los panelistas las muestran se encontraban completamente cocinadas por dentro y con una crocancia adecuada, muy similar a los cereales comerciales. En cuanto al olor no hubo puntaje negativo, por lo que se determinó que la variación de velocidad del extrusor no afectó la presencia de olor a pota en el producto final.

De los resultados estadísticos se extrae que el atributo más importante en la evaluación sensorial, analizando el factor velocidad, fue el sabor y en segundo lugar la crocancia.

La Tabla 5 muestra los resultados de las evaluaciones físicas (IE y DA) realizadas a cada muestra de snack extruido.

Tabla 5. Resultados de las evaluaciones físicas para cada muestra de snack extruido de la mezcla (MH1).

\begin{tabular}{cccc}
\hline \multicolumn{2}{c}{ Variables Independientes } & \multicolumn{2}{c}{$\begin{array}{c}\text { Variables } \\
\text { Respuesta }\end{array}$} \\
\hline Humedad de & Velocidad de & IE & DA \\
la mezcla (\%) & tornillo (RPM) & & \\
12,6 & $\mathrm{~V}_{1}=160$ & 3,11 & 76,55 \\
12,6 & $\mathrm{~V}_{2}=180$ & 2,66 & 85,04 \\
12,6 & $\mathrm{~V}_{3}=150$ & 3,36 & 66,76 \\
\hline
\end{tabular}

La Figura 5 muestra la relación que existe entre las evaluaciones físicas y la velocidad de tornillo de la extrusora.

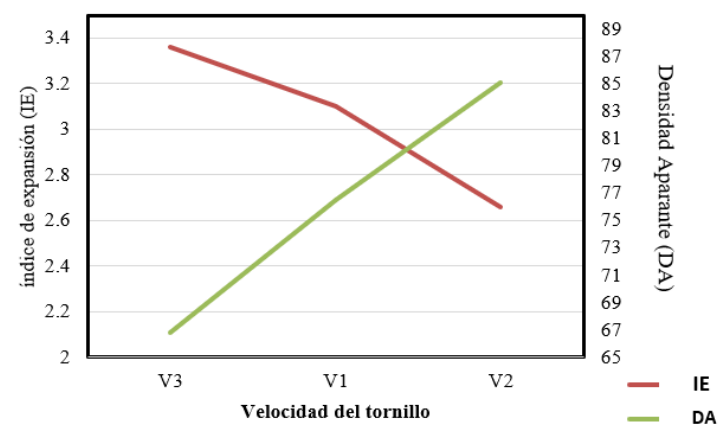

Figura 5. Relación entre las evaluaciones físicas y la velocidad de tornillo de la extrusora.

Respecto al IE, se obtiene el mayor valor para V3 y disminuye a medida que la velocidad del tornillo del extrusor disminuye, esto se debe a que a una mayor velocidad de rotación de tornillo, disminuye el tiempo de residencia y por lo tanto se genera un menor grado de cocción, además de contar con una 
mayor gradiente de velocidad y mayor intensidad de los esfuerzos de corte producidos; dicha intensidad dependerá tanto de las características propias del material (dureza, forma, distribución de las partículas etc.) como del nivel de fricción alcanzado, que a su vez depende de la presión y de la humedad. González et al. (2002) mencionan que es importante destacar que las transformaciones se producen en tiempos cortos y menores al tiempo de residencia media. La muestra V2 obtuvo un nivel de expansión menor y un DA superior al de las otras muestras, para el panel, esta muestra fue calificada como ligeramente quemada, y dura; esto debido a que, al tener una humedad relativamente baja, y una velocidad mayor, el producto produjo alta fricción del material dentro del cañón, haciendo que se queme ligeramente, y el producto salga rápidamente que no permita que su nivel de expansión logre los niveles óptimos.

Los resultados de la prueba de preferencia indicaron que la muestra de snack extruido V3 tuvo mayor grado de preferencia $(36,05 \%)$ seguido de la muestra V1 (32,33\%). Se concluyó que el snack extruido V3 elaborado con $12,6 \%$ de humedad, en la mezcla, y velocidad del tornillo de la extrusora de 150 RPM, fue el más adecuado, quedando como producto final.

\section{Determinación del tiempo de vida útil}

En la Tabla 6, se observan los valores de calidad total obtenidos aplicando el test de valoración de calidad con la escala de Karlsruhe, a muestras del extruido almacenado a la temperatura de $18^{\circ} \mathrm{C}$. A partir del valor de 5,5 (nivel de inaceptabilidad según Wittig (1981) se calculó la humedad crítica de $7,33 \%$

Tabla 6. Calidad total y humedad del extruido almacenado a temperatura de $18^{\circ} \mathrm{C}$.

\begin{tabular}{ccc}
\hline $\begin{array}{c}\text { Tiempo } \\
\text { (semanas) }\end{array}$ & $\begin{array}{c}\text { Calidad } \\
\text { total }\end{array}$ & $\begin{array}{c}\text { Humedad } \\
(\boldsymbol{\%})\end{array}$ \\
\cline { 3 - 3 } 0 & 8,7 & 5,35 \\
2 & 8,7 & 5,37 \\
3 & 8,4 & 5,49 \\
4 & 8,3 & 5,57 \\
6 & 7,6 & 6,05 \\
8 & 7,2 & 6,27 \\
10 & 6,7 & 6,55 \\
\hline
\end{tabular}

En la Figura 6 se observa que al relacionar el indicador de deterioro (humedad) vs el tiempo de almacenamiento a las diferentes temperaturas, siguen una ecuación de orden cero. Se obtuvieron las velocidades de reacción a las temperaturas estudiadas.

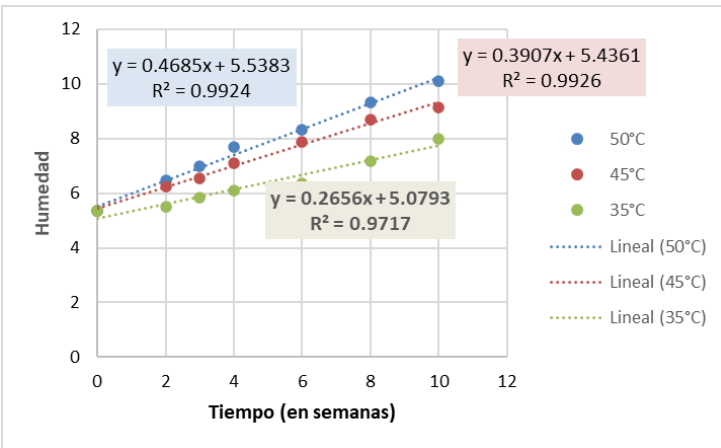

Figura 6. Variación de la humedad del snack extruido con respecto al tiempo y temperatura de almacenamiento.

Se observa una tendencia de incremento de la humedad del snack extruido en el tiempo a las temperaturas de almacenamiento. Donde a la temperatura de $50{ }^{\circ} \mathrm{C}$ la humedad aumenta en mayor proporción, debido a que con el aumento de la temperatura, las moléculas del vapor se mueven más rápido y se produce un aumento de la permeación resultando en una mayor ganancia de agua en el producto (Kilcast y Subramaniam 2000).

Con la ecuación de Arrhenius (Figura 7) se determinaron las variables cinéticas como el factor pre exponencial y la energía de activación.

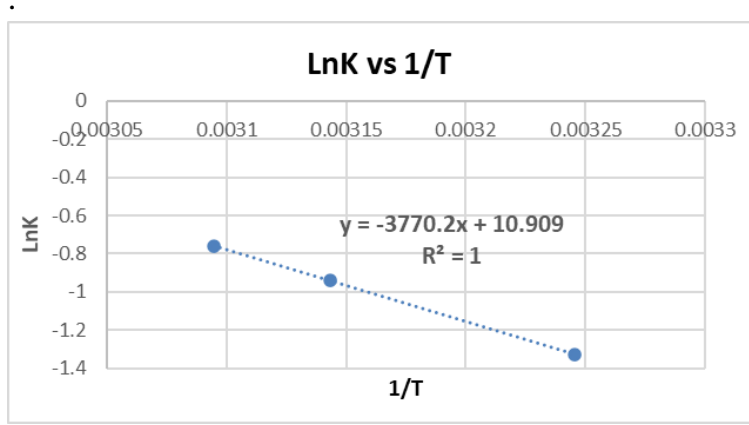

Figura 7. Ecuación de Arrhenius. 
En la Tabla 7 se muestran los parámetros utilizados para el cálculo del tiempo de vida útil, estos valores fueron reemplazados en la Ecuación 2

Tabla 7. Parámetros para el cálculo de la vida útil del snack extruido.

\begin{tabular}{cc}
\hline Parámetro & Valor \\
\hline $\mathrm{Ea}(\mathrm{Kj} / \mathrm{mol})$ & 7,487 \\
$\mathrm{~K}_{0}(\text { día })^{-1}$ & $5,46 * 10^{4}$ \\
$\mathrm{~A}_{\mathrm{o}}(\%)$ & 5,35 \\
$\mathrm{~A}(\%)$ & 7,33 \\
$\mathrm{~T}\left({ }^{\circ} \mathrm{C}\right)$ & 18 \\
$\mathrm{~T}\left({ }^{\circ} \mathrm{K}\right)$ & 291,15 \\
$\mathrm{~N}$ & 0 \\
$\mathrm{~K}_{18}$ & 0,1299 \\
\hline
\end{tabular}

Los valores hallados fueron reemplazando en la ecuación de la cinética de reacción (Ecuación 2):

$$
\begin{gathered}
\qquad \mathrm{A}=\mathrm{AO} \pm \mathrm{kt} \\
\qquad 7,33=5,35+0,1299(\mathrm{t}) \\
\text { Entonces: } \quad \mathrm{t}=15,24 \text { (semanas) }
\end{gathered}
$$

Así se obtuvo el tiempo de vida útil de 15 semanas.

\section{Análisis microbiológico del extruido final}

Los resultados microbiológicos del extruido final fueron para aerobios mesófilos $<10$. UFC/g, Mohos al igual que levaduras $<10 \mathrm{UFC} / \mathrm{g}$, Coliformes $<10$ coliformes/g, Bacillus cereus $<100 \mathrm{UFC} / g$ y no se encontró Salmonella en 25 g cumpliendo con los criterios microbiológicos según norma: RM N5912008/MINSA, considerándose apto para el consumo humano.

\section{CONCLUSIONES}

El estudio permitió obtener un snack extruido en base de maíz y arroz molidos y kiwicha complementado con concentrado de proteína de pota y leche en polvo. El producto obtenido cumplió con los requerimientos de proteínas y aminoácidos para alimentación infantil de 5 a 12 años. La mayor aceptabilidad del snack extruido se obtuvo con $4 \%$ de concentrado de proteína de pota. El producto es de forma cilíndrica y las características sensoriales más destacadas fueron la crocantes y ausencia de olor y sabor a pota. En almacenamiento utilizando bolsas de polipropileno bilaminado (BOPP) metalizado, su vida útil fue de 15 semanas. Por las características nutricionales y sensoriales obtenidas en el estudio, el producto extruido elaborado podría ser considerado en proyectos sociales de asistencia alimentaria del país.

\section{Conflictos de intereses}

Los autores firmantes del presente trabajo de investigación declaran no tener ningún potencial conflicto de interés personal o económico con otras personas $\mathrm{u}$ organizaciones que puedan influir indebidamente con el presente manuscrito.

\section{Contribuciones de los autores}

Preparación y ejecución: KE, DR, NM; Desarrollo de la metodología: KE, DR, NM; Concepción y diseño: KE, DR, NM; Edición del artículo: DR, NM; Supervisión del estudio: DR, NM.

\section{LITERATURA CITADA}

- Andersson, Y., Hedlund, B., Jonsson, L., \& Svensson. S. (1983). Extrusion cooking of a high-fiber cereal product with crispbread character. En Björck, I.; †Asp, N.he effects of extrusion cooking on nutritional value - A literature review. Journal of Food Engineering. 2(4), 281-308. Disponible en https://doi.org/10.1016/0260-8774(83)90016-X.

- AOAC. (2012). Official Methods of Analysis, Association of Official Analytical Chemist Washington D.C., Association of Official Agricultural Chemist.

- Conover, W. (1980). Practical Nonparametric Statistics. New York. John Wiley \& Sons. 493 p

- Córdova, J. (2016). Efecto del polvo protéico de pota (Dosidicus gigas) como insumo para la elaboración de yogurt. Tesis de Magister en Ciencias de los Alimentos. Universidad Nacional Mayor de San Marcos. Lima. Perú 104 p.

- Cugiño, M. (2008). Desarrollo de Alimentos Precocidos por Extrusion a Base de MaizLeguminosa. Instituto de Tecnología de los Alimentos (ITA) Facultad de Ingeniería Química. Argentina. 90 p. Disponible en: https://tecgranosysemillas.files.wordpress.com/ 2013/05/desarrollo-de-alimentos-precocidos- 
por-extrusic3b3n-a-base-de-mac3adz-

leguminosa.pdf

- Delahaye, P., Vázquez, H., Herrera, I., \& Garrido, R. (1997). Snacks de maíz enriquecidos con fibra dietética y carotenoides de la harina de zanahoria (Daucus carota) procesados por extrusión. Revista de la Facultad de Agronomía 23: 235-248.

- FAO. (1981). Contenido en aminoácidos de los alimentos y datos biológicos sobre las proteínas. Dirección de Nutrición, Servicio de Ciencia y Política de la Alimentación. Roma. 285 p. Disponible en: http://www.fao.org/docrep/005/AC854T/AC85 4T00.htm

- Fernández, J., Garcia, T., \& Martínez, R. (2009). Evaluación de la vida útil de los alimentos. Caracas, Venezuela.

- García, M. (1990). Alimentación Humana. Errores y sus consecuencias. Ed. Mundi-Prensa. Madrid. 286 p.

- González, R., Torres, R., \& De Greef, D. (2002). Extrusión-Cocción de Cereales. Boletin da Sociedade Brasileira de Ciência e Tecnologia de Alimentos. 36(2), 104-115.

- Harper, J. (1981). Effects of Extrusion Processing on Nutrients. Nutritional Evaluation of Food Processing 365-391 p. Disponible en: https://doi.org/10.1007/978-94-011-7030-7_14

- Ingram, M., Bray, D., Clark, D., Dolmon, C., Elliot, R., \& Thetcher, F. (1983). International comisión on Microbiological Specifications. "Microorganismos de los Alimentos. Técnicas de Análisis Microbiológicos". Vol. I. Segunda Edición. I Tittle Orginal: Microorganism in Foods. Ed. Acribia S. A. Zaragoza 431p

- ITINTEC. (1984). Norma técnica peruana INTINTEC 209.226. Bocaditos. Requisitos. Instituto de Investigación Tecnológico Industrial y de normas técnicas (ITINTEC). INDECOPI, Lima Perú. 9p

- INDECOPI. (2004) Norma Técnica Peruana. NTP-209.260. Alimentos cocidos de reconstitución instantánea. Papilla, requisitos. Lima. Perú 12 p

- Kameko, J. (2005). Determinación de los parámetros de extrusión de un extrusor de bajo costo para la obtención de una mezcla base para desayuno a partir de oca (Oxalis tuberosa Mol.), Olluco (Ullucus tuberosum Loz.) y Quinua (Chenopodium quinoa 83 Hill.). Tesis de Ingeniero en Industrias Alimentarias. Universidad Nacional Agraria La Molina. Lima. Perú.

- Kilcast, D., \& Subramaniam, P. (2000) The stability and shelf-life of food, published by Woodhead Publishing Limited, Abington Hall, Abington Cambridge CB1 6AH England

- Labuza, T.P. (1982). Shelf life dating of foods. Westport, Connecticut: Food \& Nutrition Press.362p 255-269.

- Lazo, L. (2006). Elaboración de harina de pota (Dosidicus gigas) precocida para consumo humano. Tesis de Ingeniero Pesquero. UNALM. Lima. Perú 150 p.

- Mercier, C., Linko, P., \& Harper, J. (1998). Extrusion Cooking. American Association of Cereal Chemists, Inc. Minnesota. 471 p.

- Madrid, A., Madrid, J., \& Madrid, R. (1994). Tecnología del pescado y productos derivados. Ed. Acribia. S.A. Zaragoza 376 p.

- Milán-Carrillo, J., Reyes-Moreno, C., CamachoHernández; I.L., \& Rouzaud-Sandez, O. (2002). Optimization of extrusion process to transform hardened chickpeas (Cicerarietinum L) into a useful product. J. Sci. Food Agric. 82, 17181728.

- Muller, H., \& Tobin, G. (1986). Nutrición y ciencia de los alimentos. Edit. Acribia S.A. Zaragoza $321 \mathrm{p}$.

- Ministerio de Salud- Digesa (2008) Resolución ministerial N591-2008/MINSA Norma sanitaria que establece los criterios microbiológicos de calidad sanitaria e inocuidad para los alimentos y bebidas de consumo humano.

https://www.saludarequipa.gob.pe/desa/archivo s/Normas_Legales/alimentos/RM591MINSAN ORMA.pdf.

- Osorio, C. (2009). Utilización de harina de pota (Dosidicus gigas) precocida en la elaboración de hojuelas extrudidas. Tesis de Ingeniero Pesquero. Universidad Nacional Agraria La Molina, Lima. Perú. 124 p. 
- Perry, J. (1980). Manual del Ingeniero Químico. Unión Tipográfica Editorial Hispano Americana. México D.F. Tomo II. 450 p.

- Roldán, D., \& Lazo, L. (2009). Características nutricionales del concentrado de proteína de calamar gigante (Dosidicus gigas) (CPLamolina)". Artículo aprobado en Anales Científico. UNALM.

- Sevatson, E., \& Huber, G. (2000). Extrusores en la industria de alimentos. En: Riaz, M. Extrusores en las aplicaciones de alimentos. Ed. Acribia S. A. Zaragoza. 181- 203 p.

- Ureña, M., D’arrigo, M., \& Girón, O. (1999). Evaluación sensorial de los alimentos, aplicación didáctica. Lima: Ed. Agraria; 197 p.

- Valls, A. (1993). El proceso de extrusión en cereales y habas de soja. Barcelona IX Curso de especialización Cotécnica FEDNA. 8 p.
- Witting, E. (1981). Evaluación sensorial, una metodología actual para tecnología de alimentos. Gráficos USACH. Santiago. 134 p.

- Yanova, M., Oleynikova, E., Sharopatova, A., \& Olentsova, J. (2019). Increasing economic efficiency of flour production from grain of the main cereal crops by extrusion method. IOP Conf. Series: Earth and Environmental Science 315, 022024. Disponible en: https://iopscience.iop.org/article/10.1088/17551315/315/2/022024/pdf

- Young, V., \& Reeds, P. (2002). Nutrición y metabolismo de proteínas y aminoácidos. En: Gibney, M.; Vorster, H.; Kok, F. Introducción a la nutrición humana. Ed. Acribia. Zaragoza. 51 87 p. 